\title{
A Proposal and Investigation of Displaying Method by Passive Touch with Electrostatic Tactile Display
}

\author{
Hirobumi Tomita ${ }^{1(\bowtie)}$, Satoshi Saga ${ }^{2}$, Shin Takahashi ${ }^{1}$, \\ and Hiroyuki Kajimoto ${ }^{3}$ \\ 1 University of Tsukuba, 1-1-1 Tennodai, Tsukuba, Ibaraki, Japan \\ tomita@iplab.cs.tsukuba.ac.jp, shin@cs.tsukuba.ac.jp \\ ${ }^{2}$ Kumamoto University, 2-39-1, Kurokami, Chuo-ku, Kumamoto, Japan \\ saga@saga-lab.org \\ 3 The University of Electro-Communications, 1-5-1, Chofugaoka, \\ Chofu, Tokyo, Japan \\ kajimoto@kaji-lab.jp
}

\begin{abstract}
In the field of tactile displays, electrostatic force displays have been developed for presenting tactile stimulation on a screen. However, with the conventional electrostatic force displays, the user cannot feel the stimulation without rubbing on the display. In this paper, to solve this problem, we propose a new method to present a tactile sensation without moving a finger by creating a small space between an electrode and an insulating surface. Moreover, we evaluate the perceived threshold of the proposed display through the experiment.
\end{abstract}

Keywords: Electrostatic tactile display $\cdot$ Passive touch $\cdot$ Conductive thread

\section{Introduction}

In the field of tactile displays, electrostatic force displays have been developed for presenting tactile stimulation on a screen. The electrostatic tactile display consists of a high-voltage generator, an electrode, and an insulator. In this method, the user touches the insulating film on the electrode. If high voltage is applied to the electrode, he/she feels the tactile stimulation by rubbing on the film. In the 1950s, Mallinckrodt et al. discovered that a phenomenon such as vibration-like friction was generated through an insulating film by electrostatic force [5]. Related surveys, dealing with parameters like the effect of input frequencies, waveforms, or amplitude modulations were conducted by many researchers $[1,3,4,7,9]$.

However, in this method, the tactile stimulation is not presented when the user stops moving the finger, and there is little research on displaying the method by passive touch with an electrostatic tactile display. Pyo et al. developed the 
tactile display, which generates electrovibration and mechanical vibration by using the electrostatic parallel plate actuator [6]. This device enables passive touch with electrostatic force, however, it requires a voltage higher than $1 \mathrm{kV}$.

In this paper, we propose a new method to present tactile sensation without moving a finger; we also implement and evaluate a device applying the proposed method. If passive tactile presentation becomes possible by this technique, the range of applications of tactile presentation using electrostatic force will be widely expanded.

\section{Implementation of Device for Passive Touch with Electrostatic Tactile Display}

The electrostatic tactile display consists of a high-voltage generator, an electrode, and an insulator. In the conventional method, an insulating film covers a flat electrode shown in Fig. 1 (a), and a user moves his/her finger on the insulator. When a high voltage is applied to the electrode, the dielectric polarization is generated in the finger. In this state, the electrode applies an attractive static force to the finger. When the user slides his/her finger on the display, s/he feels a tactile stimulation. However, when the user does not slide his/her finger on the display, s/he cannot perceive the tactile sensation.

Here, we proposed a method that employs a parallel-plate electrostatic actuator mechanism $[2,6]$ for displaying tactile stimulation to a stationary finger without significantly changing the configuration of the electrostatic tactile display. Figure 1 (b) shows the schematic sketch of the parallel-plate electrostatic actuator to generate mechanical vibration. The stationary electrode and nonstationary electrode are set in parallel. When high-voltage is applied to the stationary electrode, the attractive electrostatic force occurs on the non-stationary electrode. We considered that a passive tactile presentation was possible by adopting Pyo et al.'s method [6]. The finger is considered as the non-stationary electrode. We created a small space by using cylindrical electrodes, which have a small diameter, such as a conductive thread or wire, as shown in Fig. 1 (c, d). In this paper, we used $120 \mu \mathrm{m}$ diameter stainless steel fiber as a conductive thread. These threads were arranged lengthwise without any gaps between them on PET resin (Fig. 2 (b)). The width of these threads arranged is $25 \mathrm{~mm}$ to allow enough space for one finger. All of these threads are conductive. They are connected to the high voltage generator. Then, we covered them with a polyvinylidene chloride insulation film. This insulating film is inexpensive and easy to replace. The thickness of this insulating film is $11 \mu \mathrm{m}$.

We considered that the slight vibration of the insulating film and finger surface became possible by applying periodic high-voltage from the high-voltage generator to the cylindrical electrode, and the vibration could be perceived by the finger. The high-voltage generator shown in Fig. 2 (c) is developed by Kajimoto laboratory (The University of Electro-Communications, Tokyo). The device includes an mbed LPC1768 microcontroller, which controls the output voltage at a maximum of $600 \mathrm{~V}$ by modifying the firmware. 
(a) Conventional method

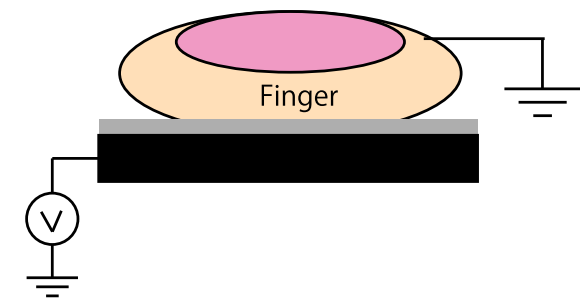

(b) Parallel plate electrostatic actuator Normal state
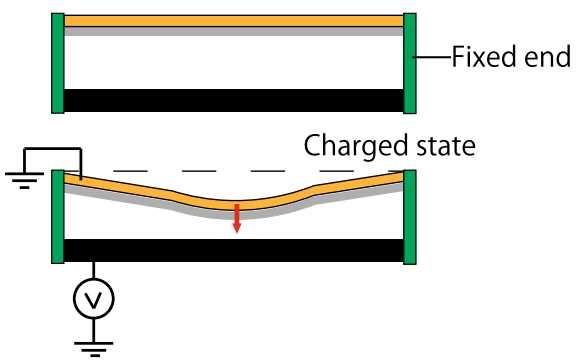

(c) Proposed method

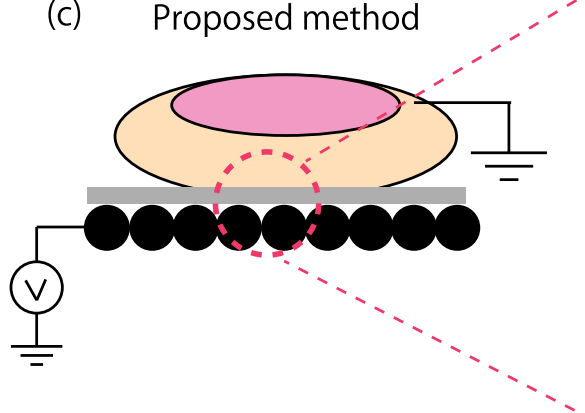

Stationary electrode
Normal state

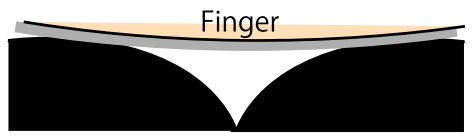

Charged state

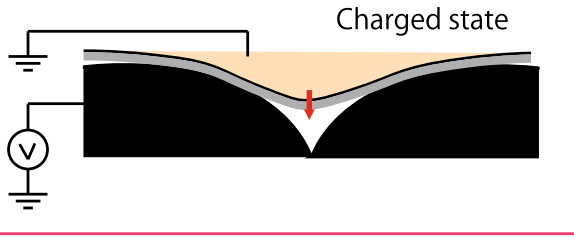

Insulator

Fig. 1. (a) Conventional electrostatic tactile display: An insulating film is placed on a flat electrode. (b) Schematic sketch of the parallel plate electrostatic actuator $[2,6]$. (c) Proposed method: A soft insulating film is placed on a nonplanar electrode, such as a conductive thread or wire. (d) Enlarged view of finger surface with the proposed method.



(a)



(b)

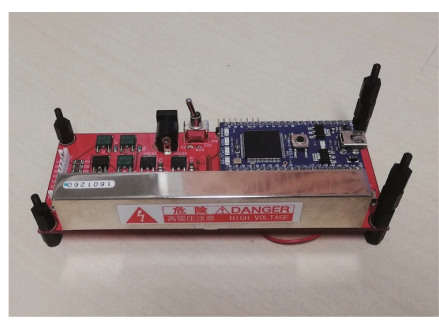

(c)

Fig. 2. (a) Overview of our display device, (b) a conductive thread arranged lengthwise and (c) the high-voltage generator. 


\section{Evaluation Experiment of Proposed Method}

We held an evaluation experiment to explore whether it is possible to feel tactile stimulation with the proposed method. Thresholds of the voltage amplitude at which the user feels the tactile sensation for various input waveforms were investigated. In this manner, we can confirm the input waveform that displays tactile sensation and how the voltage threshold changes in each input waveform.

\subsection{Evaluation Method Using Method of Constant Stimuli}

In this experiment, we investigated the perception thresholds of each voltage amplitude by using constant stimuli. As shown in Fig. 3 (a), we prepared three waveforms: sine wave, square wave, and a delta function that occurs periodically. Then we changed the voltage amplitude of these waveforms and asked the participants to answer whether they felt the stimulus presented on the tactile display. After collecting evaluation results from the participants, we calculated the perceived tactile sensation (vertical axis of Fig. 3 (b)) and psychometric function with voltage amplitude on the horizontal axis. This function is calculated by approximating the logistic curve shown in the following equation to the perceived tactile sensation in each voltage amplitude.

$$
R=\frac{1}{1+a e^{-b v}}
$$

$R$ shows the perceived tactile sensation, $v$ shows a voltage amplitude, $a$ and $b$ show constant parameters. By using this function, we estimate the threshold voltage at which the user feels the tactile sensation. In this experiment, a voltage where the psychometric function went across $50 \%$ of the perception rate was set as a threshold. An example of a psychometric function obtained from the averaged results of all participants was shown in Fig. 3 (b). By calculating the thresholds of every waveform and frequency of the input waves, we can estimate the trend of the voltage thresholds.

(a)

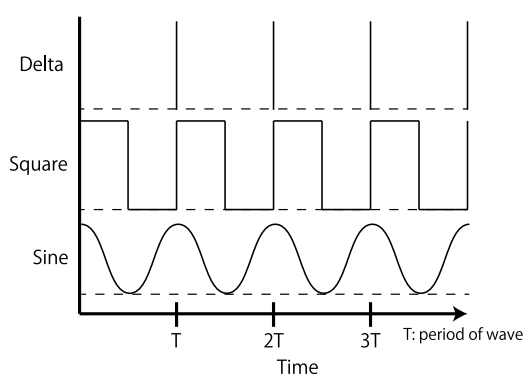

(b)



Fig. 3. (a) Three prepared waveforms and (b) an example of a psychometric function. 


\subsection{Outline of Evaluation Experiment}

We conducted an evaluation experiment of constant stimuli with 9 university students (2 female), 22 to 25 years old.

We prepared the display device, a personal computer, a polyethylene terephthalate (PET) resin stand, and a pressure sensor (FSR406) for the experiment. The display device is put on the PET resin stand to isolate it from the floor and to make it easier to place the finger. The pressure sensor is installed under the PET resin stand (Fig. 2 (a)) to measure the pressing force of the finger. We fed back the value of the pressure sensor to the participants via an LCD monitor and instructed them to keep it constant.

We prepared three waveforms and 10 types of dominant frequencies from 10 $\mathrm{Hz}$ to $630 \mathrm{~Hz}$ of each waveform. Furthermore, we prepared 9 voltage amplitudes from $200 \mathrm{~V}$ to $600 \mathrm{~V}$ for calculating the voltage threshold, and each condition was displayed ten times. These conditions were selected in random order. In addition, we investigated whether the voltage threshold changes by the difference of the pressing force of the finger. The participants were requested to perform the experiments under the conditions of a weaker pressing force (between $35 \mathrm{gf}$ and $44 \mathrm{gf}$ ) and a stronger one (between $75 \mathrm{gf}$ and $84 \mathrm{gf}$ ). From these conditions, participants conducted 5400 trials evaluation $(3$ waveforms $\times 10$ frequencies $\times$ 9 amplitudes $\times 2$ pressure $\times 10$ trials for each condition).

First of all, we explained the outline of the experiment and obtained written informed consent for participation in the study (based on ethical guidelines of University of Tsukuba) from all participants. For masking the external sound, white noise was applied to participants using a headphone during the experiment. We instructed them to place their right index finger on the display device and to keep the pressure condition during the experiment. After a waveform was inputted to the display device, participants were instructed to select either "feel something" or "feel nothing" by using the installed keyboard. After participants finished the evaluation with all the waveforms, we instructed them to keep the other pressure condition and repeat the evaluation. 5-minute breaks were taken three times during the experiment. After the experiment, we collected the participants' answers and calculated a voltage threshold for each input waveform.

\subsection{Results and Discussion}

The results of the evaluation experiment are shown in Fig. 4 and Fig. 5. The horizontal axis of these graphs shows the frequency of the input waveform. The blue line indicates the condition of a weaker pressure, and the orange line indicates the condition of a stronger pressure in these graphs. Figure 4 shows the number of participants who perceived some tactile sensation for each input waveform. Figure 5 shows the result of the voltage thresholds in each input waveform. These thresholds are derived from the intersection of the $50 \%$ line and the logistic curve. From Fig. 4 and Fig. 5, we observed that most of the participants could perceive the displayed tactile sensation in all conditions of square waves and limited frequency conditions of sine waves or delta functions. From the result, the 

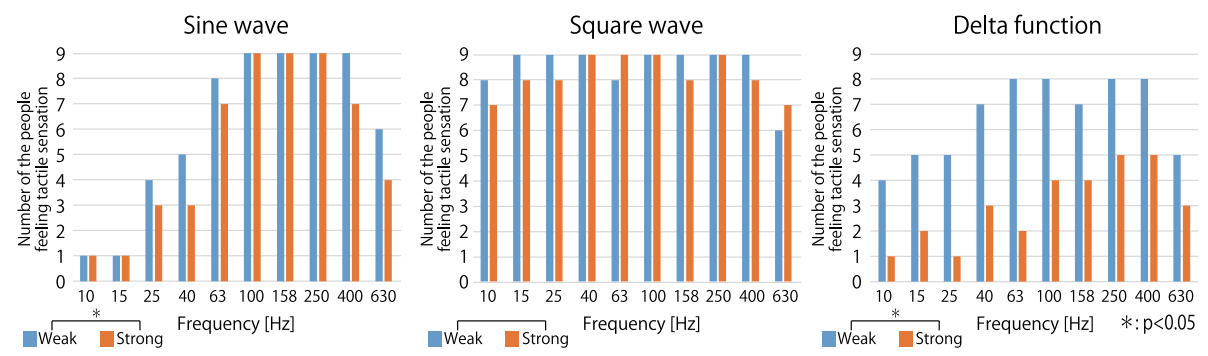

Fig. 4. Results of the number of participants who feel tactile sensation for each input waveform. (Color figure online)
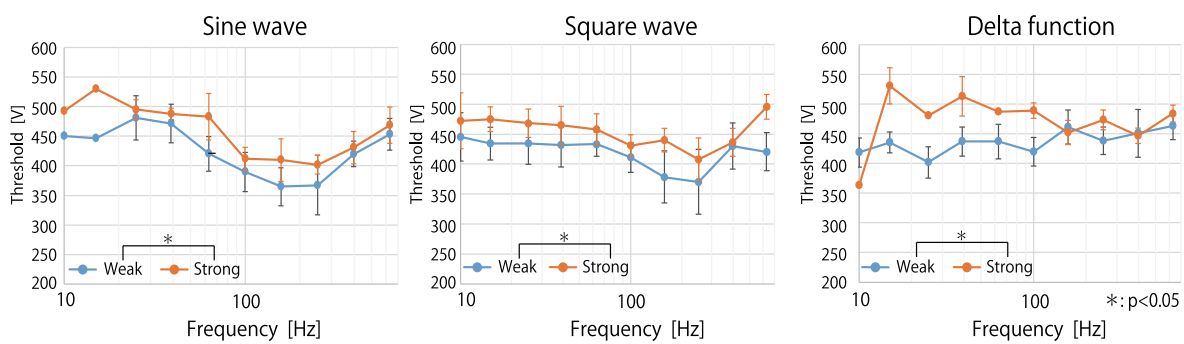

Fig. 5. Results of the threshold of voltage amplitude for each input waveform.

availability of the tactile display without moving a finger is confirmed by using our proposed method. Furthermore, the voltage thresholds are observed to be less than $500 \mathrm{~V}$ in most waveforms. In addition, we found the following two characteristics; the evaluation results differed according to the pressing force, and the outline of the graph differed according to the waveform.

Regarding the voltage thresholds, the results of $350 \mathrm{~V}$ to $500 \mathrm{~V}$ are very high compared to general tactile display devices. However, it is possible to reduce the voltage thresholds by making the insulation film thinner, and the advantage of this method is that there is almost no current flow and power at the display side. Then, it is possible to install many tactile displays or large area tactile displays. Therefore, even if a high voltage is required, we consider that this method of tactile presentation is effective for some specified applications.

Regarding the pressing force, we confirmed that the evaluation result differs depending on the pressing force. In Fig. 4, there is a significant difference in the number of participants who perceived the tactile sensation between the two pressing conditions in the sine waves and delta function. From Fig. 4, we observe that the number of participants who perceived the tactile sensation is larger under the weak pressure condition than that of under the strong pressure condition in most sine waves and delta functions. Most participants perceived the tactile sensation under both conditions in the square waves. In Fig. 5, there was a significant difference between the two pressure conditions at all waveforms. Furthermore, the averaged voltage threshold appeared to be smaller under the 
weak pressure condition than that under the strong pressure condition in all of the square waves, some sine waves and some delta function. We considered that the factor of this result is based on the ratio between the pressing force of the finger and a just noticeable difference. We focused on Weber's law and showed it in the following equation.

$$
\frac{\Delta R}{R}=\text { Const }
$$

where $\Delta R$ is a just noticeable difference in the tactile sensation, and $R$ is a pressing force in this evaluation experiment. From Eq. (2), the more pressing force, the more a difference of perceived amount is needed. Therefore, we considered that the threshold of input voltage amplitude had to be high for decreasing a just noticeable difference when the pressing force was strong.

Regarding the trend difference of graphs between waveforms (Fig.4), when square waves were displayed, most of the participants could perceive the displayed tactile sensation. However, the participants could not perceive the tactile sensation in some frequencies of sine waves and delta functions. As seen in Fig. 5, the graphs in the case of sine and square waves are downward convex with the minimum value around $200 \mathrm{~Hz}$. The graph in the case of the delta function is gentle and upward right. The reason for the shapes is considered to be related to the frequency components of each input waveform and the frequency responses of human mechanoreceptors. Vardar et al. investigated how displayed waveforms affect haptic perceptions of vibration under electrostatic tactile display [8]. Then they observed that the participants were more sensitive to square wave stimuli than sine-wave stimuli for a dominant frequency lower than $60 \mathrm{~Hz}$. Furthermore, they discussed that a low dominant frequency square wave still contains highfrequency components that stimulate the Pacinian channel. Our experimental results were similar to the results of theirs; voltage thresholds are higher in the case of sine wave stimuli than in the case of square one under $40 \mathrm{~Hz}$ frequencies. In the case of the delta function, these waves have many frequency components, however, they are smaller in amplitude than the other two waveforms. This could be because the waveforms did not have sufficient amplitude to stimulate mechanoreceptors at low dominant frequencies.

\section{Conclusions}

In this paper, we proposed a method that can display tactile stimulation without moving a finger with an electrostatic tactile display, and we conducted an evaluation experiment. The proposed method is based on the vibration of a thin insulating film, which is realized by creating a small space between the electrode and the insulator. We use a conductive thread as an electrode and place a plastic film on the conductive thread. We explored whether the user can feel tactile stimulations under several conditions. We collected the evaluation results of the participants under the conditions of waveforms, frequencies and pressing forces, and calculated the voltage threshold. From the results, the possibility of a tactile display without moving a finger is confirmed by using our proposed method. 
Besides, we also revealed the following fact: the evaluation results differed according to the pressing force and the outline of the graph differed according to the waveform. Regarding the pressing force of the finger, we considered that the ratio between the pressing force and the suction force induce the difference of perceptibility. Regarding the difference in the shapes of the graphs, we considered that the frequency components of the input waveform and the frequency responses of mechanoreceptors is the reason.

In the near future, we plan to clarify the modeling of tactile perception using our proposed method and the difference in the width of tactile expression compared with the conventional method. Further, we will perform more precise experiments to observe the physical phenomenon between the surface of the finger and the display.

\section{References}

1. Bau, O., Poupyrev, I., Israr, A., Harrison, C.: Teslatouch: electrovibration for touch surfaces. In: Proceedings of the 23Nd Annual ACM Symposium on User Interface Software and Technology, UIST 2010, pp. 283-292. ACM, New York (2010). https:// doi.org/10.1145/1866029.1866074

2. Burugupally, S.P., Perera, W.R.: Dynamics of a parallel-plate electrostatic actuator in viscous dielectric media. Sens. Actuators A: Phys. 295, 366-373 (2019)

3. Jiao, J., et al.: Detection and discrimination thresholds for haptic gratings on electrostatic tactile displays. IEEE Trans. Haptics 12(1), 34-42 (2018)

4. Kang, J., Kim, H., Choi, S., Kim, K.D., Ryu, J.: Investigation on low voltage operation of electrovibration display. IEEE Trans. Haptics 10(3), 371-381 (2016)

5. Mallinckrodt, E., Hughes, A., Sleator Jr., W.: Perception by the skin of electrically induced vibrations. Science (1953)

6. Pyo, D., Ryu, S., Kim, S.-C., Kwon, D.-S.: A new surface display for 3D haptic rendering. In: Auvray, M., Duriez, C. (eds.) EUROHAPTICS 2014. LNCS, vol. 8618, pp. 487-495. Springer, Heidelberg (2014). https://doi.org/10.1007/978-3-66244193-0_61

7. Strong, R.M., Troxel, D.E.: An electrotactile display. IEEE Trans. Man-Mach. Syst. 11(1), 72-79 (1970)

8. Vardar, Y., Güçlü, B., Basdogan, C.: Effect of waveform on tactile perception by electrovibration displayed on touch screens. IEEE Trans. Haptics 10(4), 488-499 (2017)

9. Vezzoli, E., Amberg, M., Giraud, F., Lemaire-Semail, B.: Electrovibration modeling analysis. In: Auvray, M., Duriez, C. (eds.) EUROHAPTICS 2014. LNCS, vol. 8619, pp. 369-376. Springer, Heidelberg (2014). https://doi.org/10.1007/978-3-66244196-1_45 
Open Access This chapter is licensed under the terms of the Creative Commons Attribution 4.0 International License (http://creativecommons.org/licenses/by/4.0/), which permits use, sharing, adaptation, distribution and reproduction in any medium or format, as long as you give appropriate credit to the original author(s) and the source, provide a link to the Creative Commons license and indicate if changes were made.

The images or other third party material in this chapter are included in the chapter's Creative Commons license, unless indicated otherwise in a credit line to the material. If material is not included in the chapter's Creative Commons license and your intended use is not permitted by statutory regulation or exceeds the permitted use, you will need to obtain permission directly from the copyright holder. 